\title{
Proceeding
}

9th INSHS International Christmas Sport Scientific Conference, 4-6 December 2014. International Network of Sport and Health Science. Szombathely, Hungary

\section{Effects of high-intensity strength interval training program on body composition}

\author{
MICHAELA JURÁNKOVÁ $\checkmark$, JIŘÍ BÍLÝ, EDUARD HRAZDÍRA \\ Faculty of sport studies, Masaryk Univerzity, Brno, Czech Republic
}

\begin{abstract}
Juránková, M., Bílý, J., \& Hrazdíra, A. (2015). Effects of high-intensity strength interval training program on body composition. J. Hum. Sport Exerc., 9(Proc1), pp.S314-S319. The aim of this work was to examine effects of 10-week high-intensity strength interval training (HIIT) program on body composition. Seven women ( $31.0 \pm 6.0$ years old, $65.7 \pm 9.8 \mathrm{~kg}$ body weight, $23.6 \pm 2.8 \mathrm{~kg}^{*} \mathrm{~m}-2 \mathrm{BMl}, 18.6 \pm 5.8 \mathrm{~kg}$ body fat, $26.0 \pm 3.4 \mathrm{~kg}$ muscle mass) completed intervention program. We performed an analyze of body composition before and after training program. We focused especially on body fat and muscle mass. Each session consisted of short term bouts (until $30 \mathrm{~s}$ duration) with rest in ratio 2:1. HIIT itself lasted 15-20 min three times per week (30 trainings overall). The results showed that 10-week high-intensity strength interval training have nonsignificant positive effect on decrease of body fat and significant positive effect on increase of muscle mass. This result is unconvincing and it is appropriate to continue in research with more test subjects and more homogenic sample. Key words: HIIT, TRAINING, INTERVAL, WOMEN, BODY FAT, MUSCLE MASS.
\end{abstract}

\footnotetext{
Corresponding author. Masaryk Univerzity, Kamenice 5, 62500, Brno, Czech Republic

E-mail: 259613@mail.muni.cz

9th INSHS International Christmas Sport Scientific Conference, 4-6 December 2014. International Network of Sport and

Health Science. Szombathely, Hungary.

JOURNAL OF HUMAN SPORT \& EXERCISE ISSN 1988-5202

(c) Faculty of Education. University of Alicante

doi:10.14198/jhse.2015.10.Proc1.20
} 


\section{INTRODUCTION}

High-intensity strength interval training (HIIT) is one of the training methods for changes of body composition during which there are alternated intensive short term bouts of work with time for rest.

Regular continuous endurance training is commonly used for changes in body composition, in contrast, short term bouts of HIIT are generally thought to have less of an effect on aerobic energy metabolism.

Gibala (2007) describes, that from comparation a group of subjects who performed HIIT protocol versus another group who performed 90 to 120 minutes of continuous moderate-intensity training. The total exercise volume was approximately $90 \%$ lower for the HIIT group and there was remarkably similar changes in muscle oxidative capacity and exercise capacity as describe. Naimo et al. (2015) describes similar positive effect of HIIT. He compared the traditional workout, by using moderate intensity (about 65 $\%$ of heart rate) performed for $45-60$ minutes, to HIIT and discovered, that HIIT is more effective than moderate intensity training. HIIT elicited positive effects in muscle thickness and power.

HIIT is indeed a very time-efficient training strategy. As Talanian et al. (2007) describes, only seven sessions of HIIT over 2 week induced marked increases in whole body and skeletal muscle capacity for fatty acid oxidation during exercise.

Numerous researches shows that HIIT workouts can be used to promote fat loss, increase both aerobic and anaerobic fitness, and even improve blood pressure, insulin sensitivity, and glucose regulation in a relatively short time (Olson, 2014).

Despite its lower energy cost during exercises, the HIIT program induced a more pronounced reduction in subcutaneous adiposity compared with the traditional continuous endurance exercise training program as describe Tremblay, Simoneau \& Bouchard (1994). When corrected for the energy cost of training, the decrease in the sum of six subcutaneous skinfolds induced by the HIIT program was ninefold greater than by the endurance exercise program.

In HIIT the level of metabolic rate remains elevated up to 48 hours after the training, whereas in continuous endurance training it stays increased as long as the activity lasts. HIIT for 15 minutes has greater effect on metabolism than 60 minutes continuous endurance training (Herodek, Simonović, Pavlović \& Stanković, 2014).

Researchers use different period of time for work and rest in HIIT. For example Tabata protocol is one of the most known. The protocol consists of 20 seconds of maximum intensity exercise followed by 10 seconds of rest, repeated 8 times. Tabata et al. (1996) recorded after 8 weeks of such an interval training, the anaerobic capacity was increased by $28 \%$, while the VO2 max was increased by $14 \%$.

But there exist several variants with different period of time for work and rest. Kelly, King, Goerlach \& Nimmo (2013) used $10 \times 1$ min HIIT bouts followed by 1 min rest, Boutcher (2011) used $8 \mathrm{~s} \mathrm{HIIT} \mathrm{bouts}$ followed by $12 \mathrm{~s}$ very low intensity, Lau et al. (2014) used $15 \mathrm{~s}$ intensive work with rest in ratio 1:1, Gibala (2007) used 4-7x $30 \mathrm{~s}$ HIIT bouts followed by 4 min rest.

HIIT in not appropriate only for adults, but as Lau et al. (2014) find out, HIIT is an effective and timeefficient intervention for improving of body composition, functional walking and aerobic endurance in 
overweight children (age: $10.4 \pm 0.9$ years). They performed 15 -second run at the required speed, followed by a 15 -second passive recovery for 6 weeks.

\section{MATERIAL AND METHODS}

The aim of this work was to examine effects of 10 -week high-intensity strength interval training (HIIT) program on body composition (especially on body fat and muscle mass).

The characteristics of the groups

We had a sample of 10 women from general population of Brno region $(31.0 \pm 6.0$ years old, $65.7 \pm 9.8 \mathrm{~kg}$ body weight, $23.6 \pm 2.8 \mathrm{~kg}{ }^{*} \mathrm{~m}-2 \mathrm{BMl}, 18.6 \pm 5.8 \mathrm{~kg}$ body fat, $26.0 \pm 3.4 \mathrm{~kg}$ muscle mass).

During the 10-week training process three women had to terminate this intervention. The reason for all three cases were medical complications. (Their values in the basic characteristics are not included). Training program ended 7 women. Their characteristics before HIIT program are presented in the Chart 1.

Table 1. Personal characteristics of tested women

\begin{tabular}{ccccccc}
\hline & $\mathrm{N}$ & Years & body weight & body fat & muscle mass & $\mathrm{BMI}$ \\
\hline Women & 7 & $31 \pm 6$ & $65.7 \pm 9.8 \mathrm{~kg}$ & $18.6 \pm 5.8 \mathrm{~kg}$ & $26.0 \pm 3.4 \mathrm{~kg}$ & $23.6 \pm 2.8 \mathrm{~kg}^{*} \mathrm{~m}$ \\
\hline
\end{tabular}

Training program

High intensity strength interval program took 10 weeks, three times per week (30 trainings overall). First two weeks were intervals shorter. In other session were consisted of short term bouts (until $30 \mathrm{~s}$ duration) with rest in ratio 2:1. One HIIT itself lasted 15-20 min. The whole training unit with warm up and cool down phase took to $45 \mathrm{~min}$. Tested subjects perform the warm up and stretching according to prepared scheme and were supervised by professional lector. Before the start of whole programme, there were a meeting with all participants, where we made the basic theoretical preparation and practical preparation for training unit, we eliminated undesirable elements and perform their personal analysis. In our research we can confirm high intensity for tested subjects and controlling during the training unit.

HIIT training unit was composed of several exercises which were combined with each other. The most frequently used exercises: burpee, skipping, rope skipping, squat with jump, run on H.E.A.T machine, climber, combinations of push ups, jumping lunge etc.

Methods of data collection, data processing and evaluation

The whole intervention program was conducted within specific research MUNI/A/0874/2013. Effect of endurance in selected interventions (HIIT vs. classical aerobic training vs.combined endurance and strength training) to reduce fat component, changes in resting metabolism and aerobic capacity, at Faculty of sport studies.

All women complete body analysis with Inbody 720 before the intervention and shortly after the program. Also recommendations and analysis of diet were given to all of them.

We obtained changes in body composition. The primary aim was observed changes in body fat and muscle mass. The secondary parameter was body weight. 
We processed the obtained results by mathematical-statistical and graphical methods. For processing the measured values, we used Microsoft Office Excel 2007 and statistical program STATISTICA, version 9.0, by StatSoft (Tulsa, USA). For better illustration and orderliness, the results were get into charts and graphs. The statistical significance was defined $p<0,05$.

\section{RESULTS}

Ten weeks intervention HIIT program finished 7 women. Changes in the body composition are presented in the Chart. 2. We compared values of body fat, muscle mass, weight, percentage of body fat and body mass index.

Table 2. Personal characteristic of testing women before and after intervention program

\begin{tabular}{|c|c|c|c|c|c|c|c|c|c|c|}
\hline \multirow[t]{2}{*}{ Women } & \multicolumn{2}{|c|}{ Weight (kg) } & \multicolumn{2}{|c|}{ Body fat (kg) } & \multicolumn{2}{|c|}{$\begin{array}{l}\text { Muscle mass } \\
(\mathrm{kg})\end{array}$} & \multicolumn{2}{|c|}{$\begin{array}{c}\text { Percentage of body } \\
\text { fat (\%) }\end{array}$} & \multicolumn{2}{|c|}{$\begin{array}{c}\text { Body mass index } \\
(\mathrm{kg} / \mathrm{m} 2)\end{array}$} \\
\hline & before & after & before & after & before & after & before & after & before & after \\
\hline TW1 & 75.6 & 76.9 & 20.7 & 22.3 & 30.64 & 30.33 & 27.42 & 28.98 & 25.26 & 25.69 \\
\hline TW2 & 57.3 & 57.3 & 14.1 & 13 & 23.65 & 24.35 & 24.52 & 22.71 & 19.83 & 19.86 \\
\hline TW3 & 71.4 & 71 & 22.8 & 22.4 & 26.68 & 26.77 & 31.99 & 31.53 & 24.71 & 24.57 \\
\hline TW4 & 63.4 & 67.3 & 11.7 & 12.9 & 28.69 & 30.09 & 18.48 & 19.19 & 21.31 & 22.62 \\
\hline TW5 & 53.2 & 52.6 & 14.4 & 12.2 & 21.12 & 22.03 & 27.06 & 23.25 & 22.14 & 21.89 \\
\hline TW6 & 60 & 58.2 & 17.8 & 15.3 & 23.11 & 23.59 & 29.69 & 26.33 & 24.03 & 23.31 \\
\hline TW7 & 79.1 & 77.1 & 28.4 & 25.8 & 28.09 & 28.52 & 35.92 & 33.43 & 28.03 & 27.32 \\
\hline
\end{tabular}

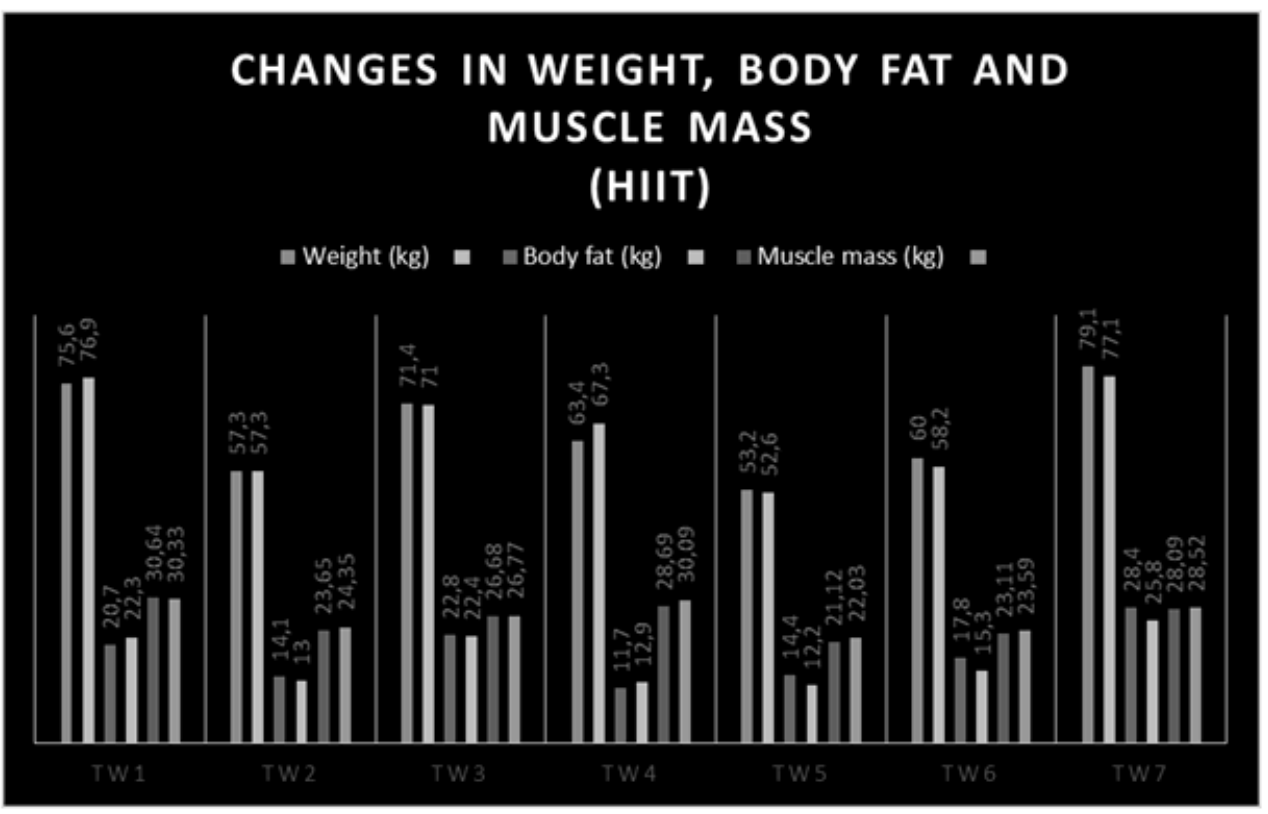

Figure 1.Changes in weight, body fat and muscle mass before and after intervence program 
The graph shows that the changes occurred were very individual. In five cases body fat decreased and muscle mass increased. We recorded decrease or steady state for body weight. On the other side there was an increasing of body fat at two women even though these women did regular exercises. We think it was caused by non-compliance to diet and psychical instability of participants.

Table 3. Wilcoxon Matched Pairs Test for body fat

\begin{tabular}{ccccc}
\hline Pair of Variables & Valid N & $\mathrm{T}$ & $\mathrm{Z}$ & $p$-value \\
\hline Fat before $(\mathrm{kg})$ \& Fat after $(\mathrm{kg})$ & 7 & 7 & 1.1832 & 0.2367 \\
\hline Wilcoxon Matched Pairs Test $\left(\mathrm{X} 1 \_S p r e a d s h e e t 3\right)$ & Marked tests are significant at $p<.05000$
\end{tabular}

Under Wilcoxon Matched Pairs Test for body fat (Chart 3) by $p<.05000$ were changes in area of body fat statisticaly nonsignificant, although at 5 tested women body fat decreased.

Table 4. Wilcoxon Matched Pairs Test for muscle mass

\begin{tabular}{ccccc}
\hline Pair of Variables & Valid N & T & Z & $p$-value \\
\hline Muscle mass before (kg)\& Muscle mass after (kg) & 7 & 2 & 2.0228 & 0.0425 \\
\hline Wilcoxon Matched Pairs Test (X1_Spreadsheet3) Marked tests are significant at & $p<.05000$
\end{tabular}

In the case of muscle mass (Chart 4.), increase was recorded at six tested women. Despite of small testing sample, difference in amount of muscle mass reach statistically significant values when $p=0.042523$.

Table 5. Wilcoxon Matched Pairs Test for weight

\begin{tabular}{ccccc}
\hline Pair of Variables & Valid N & T & Z & $p$-value \\
\hline Weight before $(\mathrm{kg})$ \& Weight after $(\mathrm{kg})$ & 6 & 9 & 0.3145 & 0.7532 \\
\hline Wilcoxon Matched Pairs Test (X1_Spreadsheet3) & Marked tests are significant at $p<, 05000$
\end{tabular}

The last observed field was body weight (Chart 5.). This parameter led to reduction followed by decrease of body fat and increase of muscle mass at four women (TW3, TW5, TW6,TW7). In two cases increase of body weight with relation to higher body fat was recorded (TW1, TW4). For the last tested woman weight stay unchanged, but we could saw increase of muscle mass and decrease of body fat (TW2). Chart n.4 shows that comparison of body weight before and after intervention programme did not proof significant changes.

\section{DISCUSSION}

The aim of this research was to reduce fat reserves using the intervention HIIT programme. Changes in primary observed parameter of body composition - body fat area, were not statistically significant. This fact we can partly explain by small tested group, when three women cannot finish the intervention because of medical issues. The number of tested persons in HIIT group we are considering as the main negative aspect of this part research.

Although the individual training units were physically very difficult, there is one interesting fact - there were several women who did not achieve the reduction of body fat but its increase. We suppose these negative changes were caused by failure to keep nutrition and dietary recommendations, which were provided by 
nutritionist at the beginning of the intervention. It is necessary to obtain bigger testing sample of women and take care more for dietary plan for continuing the research.

Intervention programs are known for their higher demands on time and subjects despite to theirs working activities were trying for regular participation in the prepared programme. Intervention compliance prosper thanks to motivation programs, financial resources and sport psychology of lectors.

\section{CONCLUSIONS}

Ten weeks high intensity intervention program did not show significant changes in observed parts such as reduction of body fat and secondary weight level of body. Only one area where significant change was proved it was increase of muscle mass. Effect of this training type is therefore very relevant. Despite of well known studies such as e.g. Izumi Tabata etc. we cannot assess if it has positive effect for weight reduction from fat reserves. We also have to consider its high difficulty and readiness of research samples. According to their feedback this type of training unit was very physically exhausting and also psychically in first few weeks. Low time intensity is taken as a positive phenomenon of this type of training unit and it is dependent on warm up as well as on cool down phase, so we can speak again about minimally 45 min of training unit. Definitely we should know all medical complications and do personal anamnesis at chosen subjects, ideally including cardiovascular load testing.

This result is unconvincing and it is appropriate to continue in research with more test subjects and more homogenous sample.

\section{REFERENCES}

1. Boutcher, S.H. (2011). High-Intensity Intermittent Exercise and Fat Loss. J Obes, 868305.

2. Lau, P.W., Wong, P., Ngo, J.K., Liang, Y., Kim, C.G., \& Kim, H.S. (2014). Effects of high-intensity intermittent running exercise in overweight children. Eur J Sport Sci, 11, pp.1-9.

3. Gibala, M.J. (2007). High-intensity interval training: a time-efficient strategy for health promotion? Curr Sports Med Rep, 6(4), pp.211-213.

4. Herodek, K., Simonović, C., Pavlović, V. \& Stanković, R. (2014). HIGH INTENSITY INTERVAL TRAINING. Activities in Physical Education and Sport, 4(2), pp.205-207.

5. Kelly, B., King, J.A., Goerlach, J. \& Nimmo, M.A. (2013). The impact of high-intensity intermittent exercise on resting metabolic rate in healthy males. Eur J Appl Physiol, 113(12), pp.3039-47.

6. Naimo, M. A., De Souza, E.O., Wilson, J.M., Carpenter, A.L., Gilchrist, P., Lowery, R.P., ... Joy, J. (2015). High-intensity Interval Training Has Positive Effects on Performance In Ice Hockey Players. Int J Sports Med, 36(1), pp.61-66.

7. Olson, M. (2014). TABATA: It's a HIIT! ACSM'S Health \& Fitness Journal, 18(5), pp.17-24.

8. Tabata, I., Nishimura, K., Kouzaki, M., Hirai, Y., Oqita, F., Miyachi, M., \& Yamamoto, K. (1996). Effects of moderate-intensity endurance and high-intensity intermittent training on anaerobic capacity and VO2max. Med Sci Sports Exerc, 28(10), pp.1327-1330.

9. Talanian, J. L., Galloway, S.D., Heigenhauser, G.J., Bonen, A., \& Spriet, L.L. (2007). Two weeks of high-intensity aerobic interval training increases the capacity for fat oxidation during exercise in women. J Appl Physiol, 102(4), pp.1439-1447.

10. Tremblay, A., Simoneau, J.A. \& Bouchard, C. (1994). Impact of exercise intensity on body fatness and skeletal muscle metabolism. Metabolism, 43(7), pp.814-818. 